\title{
Desenvolvimento e implementação do ciclo diurno da queima de biomassa no PREP-CHEM-SRC
}

\section{Development and implementation of fire diurnal cycle in PREP-CHEM-SRC}

\author{
Paula Resende Santos ${ }^{* 1} \bowtie\left(D\right.$, Gabriel Pereira ${ }^{1,2} \varangle$ (D), Francielle da Silva Cardozo ${ }^{2} \bowtie(D$, \\ Elisabete Caria Moraes ${ }^{3} \bowtie\left(D\right.$, Guilherme Augusto Verola Mataveli ${ }^{3} \bowtie$ (I) \\ ${ }^{1}$ Faculdade de Filosofia, Letras e Ciências Humanas, Universidade São Paulo, \\ São Paulo, Minas, Brasil \\ 2Departamento de Geociências, Universidade Federal de São João Del Rei, \\ Colônia do Bengo, Minas Gerais, Brasil \\ ${ }^{3}$ Coordenação-Geral de Observação da Terra, Instituto Nacional de Pesquisas Espaciais, \\ São José dos Campos, São Paulo, Brasil \\ E-mail: pereira@ufsj.edu.br (GP); franciellecardozo@ufsj.edu.br (FSC); \\ guilhermemataveli@gmail.com (GAVM); bete@ltid.inpe.br (ECM) \\ *E-mail para correspondência: paula.resende@usp.br
}

Recebido (Received): 28/08/2020 Aceito (Accepted): 21/04/2021

Resumo: Os maiores registros de queimadas no mundo estão associados as regiões tropicais, que emitem anualmente para atmosfera gases e partículas que podem de maneira direta ou indireta afetar o clima e a qualidade do ar. O objetivo desse trabalho consistiu em desenvolver uma metodologia acoplada ao modelo 3BEM_FRP que utiliza informações do tempo de duração das queimadas para diferentes tipos de uso e cobertura da terra para biomas da América do Sul com a finalidade de fornecer informações para a estimativa da Energia Radiativa do Fogo quando houver ausência de dados de Potência Radiativa do Fogo. O tempo médio de duração das queimadas para os diferentes usos e cobertura da terra do IGBP para a América do Sul foi estimado utilizando o produto WFAABA/GOES para a série temporal de 1997-2015 e implementado no modelo 3BEM_FRP. Utilizando o 3BEM_FRP acrescido do ciclo (C3BEM_FRP) foram geradas estimativas das emissões de PM2.5 para a América do Sul de 2002-2020 com a finalidade de verificar a distribuição espacial das emissões oriundas da queima de biomassa. Cerca de $61 \%$ das queimadas na América do Sul duram em média entre 6 e 7 horas e dentre os países da América do Sul, o Brasil é o país que mais emite PM2.5 oriundos da queima de biomassa, correspondendo a 58\% das emissões totais (2003-2020). O Pará foi o estado brasileiro com as maiores emissões associadas, emitindo em média 1,2 Tg PM2.5 ano ${ }^{-1}$.

Palavras-chaves: Queimadas; Emissões; 3BEM_FRP.

Abstract: The largest fires records in the world are associated with tropical regions, which can release into the atmosphere an amount of gases and particles that can be affect directly or indirectly the climate and the air quality. This work aims to develop a methodology coupled to the 3BEM_FRP model that uses fire duration information for different types of land use and land cover for biomes of South America in order to provide information for the estimation of Fire Radiative Energy when there is absence of Fire Radiative Power data. The fires mean time for the different uses and land cover type from IGBP for America was estimated using the WFAABA / GOES product from 1997 to 2015 and implemented in the 3BEM_FRP model. PM2.5 emissions was estimated for South America during 2003-2015 using the 3BEM_FRP plus cycle (C3BEM_FRP) with the purpose to verify the biomass burning emissions spatial distribution. About $61 \%$ of biomass burning in South America lasting between 6 and 7 hours and Brazil is the country that most emits PM2.5 from biomass burning, corresponding to 58\% of the emissions (2003-2020). Para was the

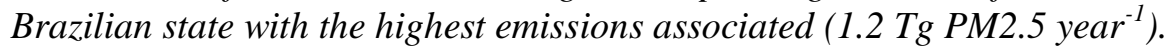

Keywords: Biomass burning; Emissions; 3BEM_FRP. 


\section{Introdução}

A queima de biomassa é um fenômeno global e desempenha um papel fundamental na dinâmica terrestre e atmosférica. Estima-se que aproximadamente 423 milhões de hectares são queimados anualmente no mundo (GIGLIO et al., 2018) e que cerca de $7115 \pm 332 \mathrm{Tg}^{\mathrm{ano}}{ }^{-1}$ de Dióxido de Carbono (CO2) são emitidos pela queima de biomassa (PROSPERI et al., 2020). O fogo é amplamente utilizado como instrumento de manejo para estimular a rebrota de forragem para a pecuária, diminuir as pragas, remover os remanescentes agrícolas e está associado a mudanças do uso e cobertura da terra, principalmente nas regiões tropicais(COCHRANE, 2009; FANIN; WERF; VAN DER, 2015; ICHOKU et al., 2016).

Nas regiões tropicais, a variabilidade interanual das queimadas está relacionada principalmente com as variáveis climáticas, uma vez que o regime hidrológico influencia a quantidade de combustível disponível para queima, a intensidade e a duração da queimada (CHEN et al., 2013). Entretanto, a inflamabilidade florestal tem aumentado devido às atividades humanas como, por exemplo, a extração seletiva de madeira, fragmentação florestal e a frequência de queimadas em uma determinada área (CARMENTA et al., 2016).

Os impactos provocados pelas queimadas não se restringem apenas às áreas atingidas pelo fogo, mas também se estendem a poluição provocada pela fumaça emitida para outras regiões distantes do foco através do transporte atmosférico (ICHOKU et al., 2016). As emissões resultantes da queima de biomassa são compostas por uma mistura de gases e partículas que podem de maneira direta ou indireta afetar o clima e a qualidade do ar. Nem toda combustão originada pela queima de biomassa é completa, emitindo além de $\mathrm{CO}_{2}$, o Monóxido de Carbono (CO), o Metano $\left(\mathrm{CH}_{4}\right)$, Compostos Orgânicos Voláteis (COVs) e materiais particulados (em inglês, total particulate matter - TPM), que estão relacionados à fuligem que afeta diretamente a saúde humana, ocasionando doenças que acometem o aparelho respiratório. A partir da formação destes compostos, há a reação dos COVs com outras substâncias presentes na atmosfera e ocorre a formação de um dos gases responsáveis pelo efeito estufa, o ozônio $\left(\mathrm{O}_{3}\right.$ troposférico) (GILMAN et al., 2015; HEILMAN et al., 2014; REDDINGTON et al., 2015; SOMMERS; LOEHMAN; HARDY, 2014)

Para modelar os impactos das queimadas na qualidade do ar e realizar uma análise confiável dos efeitos associados, é necessário que as estimativas tenham uma boa acurácia espaço-temporal e estimativas consistentes da massa total emitida (PEREIRA et al., 2016). Os dados estimados por sensores orbitais representam a principal fonte de informação para mapear as atividades de queima de biomassa e avaliar as emissões resultantes desse processo em escalas regionais e globais a partir da aquisição sistemática de dados (BOWMAN et al., 2010).

Vários inventários de emissões baseados na contagem de focos de calor durante a passagem do satélite e/ou na área queimada foram desenvolvidos nas últimas décadas, tal como o Global Fire Emissions Database - GFED (WERF et al., 2017), FLAMBE (REID et al., 2009), Fire Inventory from NCAR - FINN (WIEDINMYER et al., 2010) e Brazilian Biomass Burning Emission Model - 3BEM (Longo et al., 2010) e, recentemente, têm-se utilizado a Potência Radiativa do fogo (em inglês, Fire Radiative Power - FRP) e a Energia Radiativa do Fogo (em inglês, Fire Radiative Energy - FRE) como dado de entrada em modelos como o 3BEM_FRP (PEREIRA et al., 2009) e Global Fire Assimilation System - GFAS (KAISER et al., 2012), com a finalidade de se obter as emissões provenientes da queima de biomassa com menor incerteza associada.

A FRP corresponde a taxa de energia liberada por uma queimada e está diretamente relacionada ao consumo de combustível durante a queima. Dada a natureza discreta da aquisição dos dados de sensoriamento remoto, somente a FRP pode ser estimada diretamente pelo sensor, enquanto que a FRE pode ser estimada integrando sucessivas medições de FRP durante um determinado período de tempo (ICHOKU, KAHN; CHIN, 2012).

O sensor Moderate Resolution Imaging Spectroradiometer (MODIS), lançado em 1999 a bordo da plataforma Terra, foi o primeiro sensor orbital a estimar FRP. Posteriormente, a FRP passou a ser estimada por sensores geoestacionários, incluindo o Meteosat- SEVIRI e GOES - Imager (ICHOKU, KAHN; CHIN, 2012). As taxas de amostragem dos sensores orbitais não são suficientes para caracterizar completamente como as queimadas variam ao longo do curso do dia. Observações com uma resolução temporal muito maior estão disponíveis a partir de satélites geoestacionários, mas possuem limitações em sua resolução espacial, o que resulta em uma falha na detecção de queimadas de baixa intensidade. Devido a estas limitações, atuais inventários de emissões globais, quase em tempo real, ainda ignoram os possíveis efeitos do ciclo diurno do fogo sobre sua emissão.

Como alternativa a estas limitações, as observações do ciclo diurno das queimadas oriundas de satélites geoestacionários podem ser combinadas com a FRP estimada por diversos sistemas de satélites orbitais com a finalidade de gerar estimativas mais confiáveis. Deste modo, o objetivo deste trabalho é desenvolver uma 
metodologia acoplada ao modelo 3BEM_FRP que utiliza informações do tempo de duração das queimadas para diferentes tipos de uso e cobertura da terra para biomas da América do Sul com a finalidade de fornecer informações para a estimativa da FRE quando houver ausência de dados de FRP.

\section{2. Área de estudo}

A área de estudo corresponde ao continente Sul-Americano e suas configurações de biomas propostos pelo IBGE e Olson et al. (2001) para o Brasil e demais países, respectivamente (Figura 1). A América do Sul é formada por 15 países, e dada a sua extensão territorial há uma grande diversidade climática e biológica, cujas florestas ocupam aproximadamente $22 \%$ do seu território, representando cerca de $27 \%$ da cobertura florestal mundial.

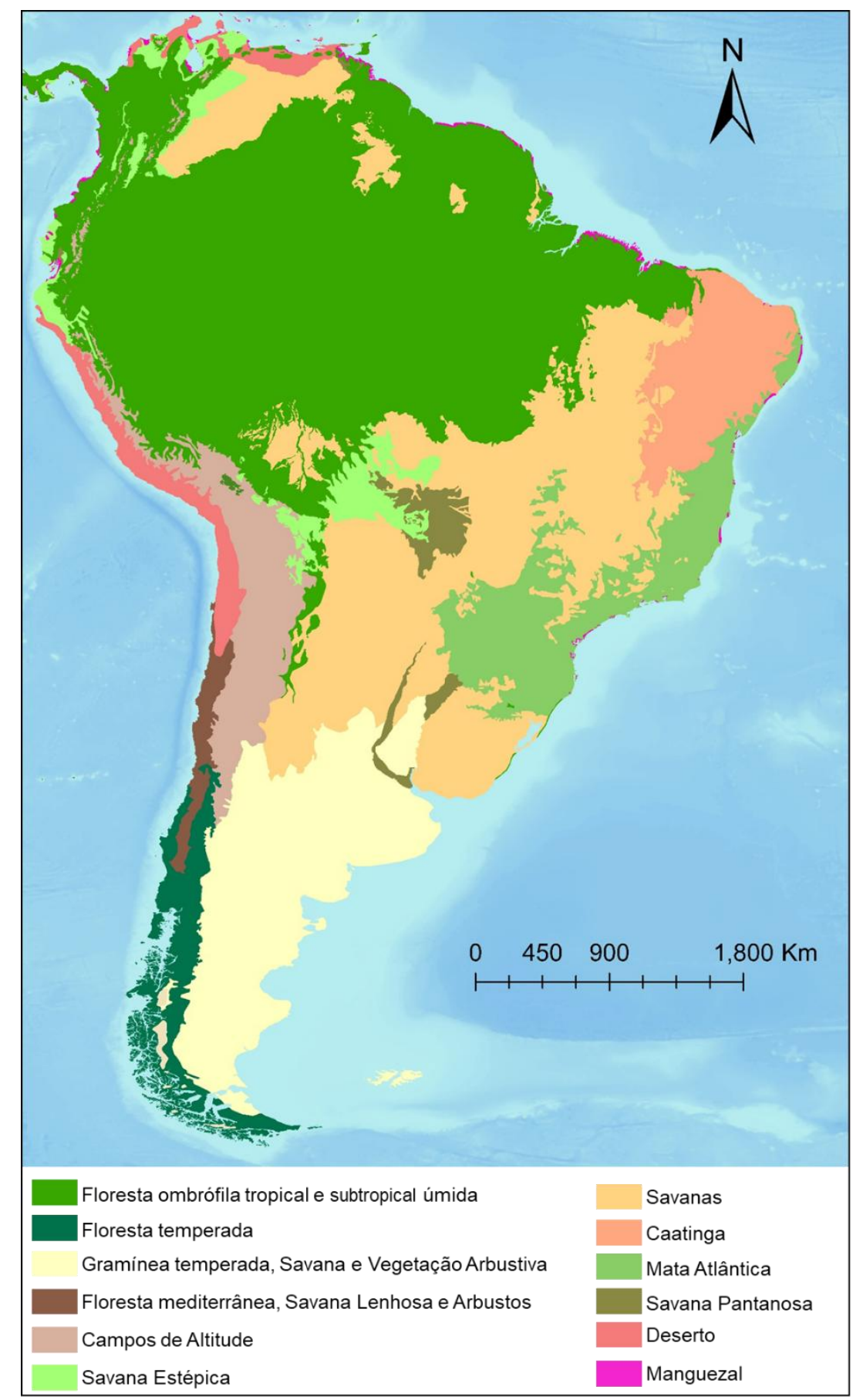

Figura 1: América do Sul e suas configurações de biomas propostos pelo IBGE e Olson et al. (2001) para o Brasil e demais países, respectivamente. 
A heterogeneidade climática dessa região provém da atuação e o desenvolvimento de diferentes sistemas atmosféricos, condicionados pelas variações na latitude e formas de relevo variadas (REBOITA $e t$ al., 2010). Durante o inverno na América do Sul, os ventos alísios oriundos do Oceano Atlântico, na região nordeste do Brasil, transportam os poluentes emitidos pelas queimadas para oeste, que encontram a barreira topográfica da Cordilheira dos Andes. Os fluxos de ar que transportam os poluentes são forçados a deslocar para a região sul e sudoeste ao encontrarem a barreira topográfica dos Andes, direcionando a fumaça para a Bolívia, Paraguai e Argentina, voltando-se para a direção do Oceano Atlântico. As correntes frias oriundas da região sul e sudoeste do continente fazem com que a massa de ar poluído retorne para região norte, passando pelas regiões mais populosas do Brasil. Este trajeto percorrido pela fumaça traz grandes implicações a população residente nestas áreas, pois estes gases somam-se a poluentes oriundos das atividades industriais e contribuem para as mudanças climáticas em diferentes escalas (FREITAS et al., 2005).

\section{Materiais e métodos}

\subsection{GOES/ WFABBA}

O WFABBA é resultado de uma parceria entre a NOAA/ NESDIS/ STAR e UW-CIMSS, desenvolvido na University of Wisconsin (UW-Madison) com a finalidade de utilizar dados de satélites geoestacionários para detectar e caracterizar a queima de biomassa. O algoritmo utiliza o sensor multiespectral Imager a bordo da série de satélites GOES, e fornece produtos a cada meia hora para o hemisfério ocidental com uma resolução espacial nominal de 4 x $4 \mathrm{~km}$ no NADIR. O sensor possui cinco bandas que cobrem o espectro eletromagnético do visível ao infravermelho termal, no entanto, o algoritmo utiliza bandas posicionadas no visível (quando disponível durante o dia), no infravermelho médio $(3,9 \mu \mathrm{m})$ e infravermelho de ondas longas $(10,7 \mu \mathrm{m})$ para estimar em tempo real a localização de focos e queimadas e sua FRP desde 2000 (XU et al., 2010).

O WFABBA é um algoritmo contextual que utiliza os pixels vizinhos para identificar os pixels candidatos a focos de queimadas através de técnicas estatísticas, tais como média, desvio padrão e aproximações de histograma. Além disso, o algoritmo utiliza máscaras em regiões de deserto, costeiras e outras regiões que possuem alta reflectância a fim de eliminar falsas detecções (KOLTUNOV, USTIN; PRINS, 2012). Assim como os produtos de FRP dos sensores MODIS e SEVIRI, a FRP estimada através dos dados providos pelo sensor Imager é obtida através do método proposto por Wooster et al. (2003) (Equação 1), em que $A_{\text {pix }}$ é a área do pixel do GOES (que varia em função do ângulo de varredura), $\sigma$ é a constante de Stefan-Boltzmann $\left(5,6704 \times 10^{-8} \mathrm{~W} \mathrm{~m}^{-2} \mathrm{~K}^{-4}\right), a$ é uma constante específica para cada sensor, que no caso do GOES é 3,08 $10^{-9}$ $\mathrm{W} \mathrm{m} \mathrm{mr}^{-1} \mu \mathrm{m}^{-1} \mathrm{~K}^{-4}$ (WOOSTER et al., 2005).

$$
F R P \approx \frac{A_{p i x} \sigma}{a t_{4}}\left(l_{4}-\bar{l}_{b 4}\right)
$$

Eq.1

Os dados deste produto informam o ângulo de visada do satélite, a latitude, longitude, o tamanho do pixel, a temperatura de brilho, a FRP, o ecossistema associado ao foco de queimada e as "fire flags". As "fire flags" são definidas como: ("0") pixel de queimada processado, ("1") pixel saturado, ("2") pixel contaminado por nuvem, ("3”) alta probabilidade de pixel que contenha uma queimada, ("4") média probabilidade de pixel que contenha uma queimada, ("5") baixa probabilidade que o pixel contenha uma queimada (categoria representa o maior número de falsas detecções) (KOLTUNOV, USTIN; PRINS, 2012).

Embora o produto WFABBA possua uma alta frequência de observações, aproximadamente $5-10 \%$ dos focos detectados pelo sensor saturam quando o tamanho e temperatura do fogo superaram a sensibilidade do sensor próximo à banda posicionada em $3.9 \mu \mathrm{m}$, inviabilizando a estimativa de FRP (PEREIRA et al., 2009).

\subsection{MCD12Q1}

O produto global MODIS Land Cover Type Product (MCD12Q1) é produzido com dados oriundos dos satélites Aqua e Terra em uma resolução espacial de 500 m e está disponível de 2001 a 2013 na coleção 5. Com a finalidade de gerar informações relacionadas ao estado atual ou a dinâmica do uso e cobertura da terra, o produto utiliza um conjunto de 17 classes desenvolvidos pelo International Geosphere-Biosphere Programme Data and Information System (IGBP-DIS) (Loveland e Belward, 1997), descritas na Tabela 1 (FRIEDL et al., 2002). 
Tabela 1: Classes IGBP-DIS do produto MCD12Q1

Classes

Hidrografia

Floresta de

Coníferas

Floresta Ombrófila densa Coníferas decidual Floresta Estacional
Decidual

Mosaico de Floresta e pastagem

Vegetação

Arbustiva Fechada

Vegetação

Arbustiva Aberta

Savana Lenhosa

Savana

Gramíneas

Permanentemente

Predominância Agrícolas

Áreas urbanas

Mosaico de áreas agrícolas/ vegetação

Gelo

Solo Exposto ou vegetação Rala
Floresta de

Áreas Alagadas

\section{Classificação IGBP}

Oceanos, mares, lagos, reservatórios e rios. Podem ser de água doce ou de água salgada.

Predominância de vegetação Aciculifoliada com altura superior a 2 m e cobertura arbórea > $60 \%$. A maioria das árvores permanecem verdes durante todo o ano. O dossel florestal sempre possui folhagem verde.

Predominânica de vegetação latifoliadas com altura superior a $2 \mathrm{~m}$ e cobertura arbórea $>60 \%$.

A maioria das árvores e arbustos permanecem verdes todo o ano. O dossel florestal sempre possui folhagem verde.

Predominância de vegetação lenhosa com altura superior a 2 m e cobertura arbórea $>60 \%$. Consiste em comunidades sazonais de árvores aciculifoliadas, com um ciclo anual de períodos com ausência de folhas.

Predominância de vegetação lenhosa com altura superior a 2 m e cobertura arbórea $>60 \%$.

Consiste em comunidades sazonais de árvores latifoliadas, com um ciclo anual de períodos com ausência de folhas.

Predominância de árvores com altura superior a $2 \mathrm{~m}$ e cobertura arbórea $>60 \%$. Consiste em comunidades de árvores intercaladas com mosaicos de vegetação arbustiva fechada e aberta, savana, savana lenhosa e gramíneas.

Áreas com vegetação lenhosa com menos de $2 \mathrm{~m}$ de altura e corbetura arbustiva $>60 \%$. A folhagem pode ser de folhas verdes ou de folha caduca.

Áreas com vegetação lenhosa com menos de $2 \mathrm{~m}$ de altura e com coberturas arbustiva variando entre $10 \%$ e $60 \%$. A folhagem pode ser de folhas verdes ou de folhas caducas.

Áreas de vegetação herbácea e de florestas com cobertura arbórea variando entre $30 \%$ e $60 \%$. A altura da floresta pode exceder $2 \mathrm{~m}$.

Áreas de vegetação herbácea e de florestas com cobertura arbórea variando entre $10 \%$ e $30 \%$. A altura da floresta pode exceder $2 \mathrm{~m}$.

Predominânia de vegetação herbácea. A cobertura de árvores e arbustos é inferior a 10\%.

Áreas com a presença permanente de água e vegetação herbácea ou lenhosa. A vegetação pode estar presente em água salgada, salobre ou doce.

áreas cobertas com culturas temporárias seguidas de colheita e um período de solo exposto.

Áreas cobertas pela macha urbana.

Áreas com um mosaico de terras cultivadas, florestas, mata e pastagens. Nenhum componente compreende mais de $60 \%$ da paisagem.

Áreas sob cobertura de neve / gelo ao longo do ano.

Áreas com solo exposto, areia, rochas ou neve. Estas áreas nunca têm mais de $10 \%$ de cobertura vegetal durante qualquer época do ano.

Fonte: Adaptada de Friedl et al. (2002).

O produto MCD12Q1 é gerado através de um algoritmo de classificação supervisionada, que utiliza a composição de 8 dias de dados de ambos os sensores MODIS. Este algoritmo apoia-se em um banco de dados de treinamento que foi desenvolvido utilizando imagens de alta resolução em conjunto com dados auxiliares. Também é fornecido ao classificador métricas anuais dos dados de reflectância bidirecional e temperatura da superfície (MODIS Land Cover Product User Guide, 2013). 


\subsection{Procedimentos metodológicos}

A seguir serão abordados os procedimentos metodológicos adotados neste trabalho, como o processamento dos dados GOES, os métodos utilizados na estimativa do ciclo diurno das queimadas e a implementação do ciclo diurno das queimadas no modelo C3BEM_FRP.

\subsubsection{Reclassificação IGBP e Pré Processamento GOES}

Com a finalidade de analisar o ciclo diurno das queimadas por tipo de uso e cobertura da terra para cada bioma, as classes do IGBP do produto MCD12Q1 foram reclassificadas para a série temporal de 2001 a 2013 com uma resolução de $0,072^{\circ}$. Deste modo, os identificadores das classes do IGBP passaram a variar de acordo com o bioma.

Os dados WFABBA disponibilizados em formato ASCII foram processados com a finalidade de eliminar pixels de baixa confiabilidade (flag=5). Nesta etapa também foram corrigidos os pixels saturados $($ flag $=1$ ) utilizando método proposto por (Pereira, 2013), uma vez que remover todos esses pixels pode fazer com que importantes eventos de queima de biomassa sejam ignorados. O método proposto é fundamentado na premissa que a radiância espectral emitida na banda centrada em 3,9 $\mu \mathrm{m}$ (MIR) é linearmente proporcional à FRP emitida $\left(F R P_{M I R}\right)$. A radiância espectral emitida $\left(M_{\lambda}\right)$ é estimada através da Equação de Planck (Equação 2), onde $C_{1}$ e $C_{2}$ são constantes $\left(3,74 \times 10^{8} \mathrm{~W} \cdot \mathrm{m}^{-2}\right.$ e $1,44 \times 10^{4} \mu \mathrm{m} . \mathrm{K}$, respectivamente); $\lambda$ é o comprimento de onda $(\mu \mathrm{m}), T$ é a temperatura em Kelvin;

$$
\begin{array}{r}
M(\lambda, T)=\frac{1}{\lambda^{5}} \times \frac{C_{1}}{e^{\left(\frac{C_{2}}{\lambda, T}\right)}-1} \\
F R P_{M I R}=\frac{A g}{a} \sigma \int_{3,76}^{4,03} M(\lambda, T) d \lambda-M_{b}
\end{array}
$$

Eq. 2

Os valores da $M_{\lambda}$ são substituídos na Equação 3 que $A g$ representa a área do pixel do sensor Imager/Goes; $a$ é a constante baseada na resposta espectral na banda referente ao MIR disponível em Wooster et al. (2005); $M_{b}$ é a emitância radiante para o background, integrada nos comprimentos de onda da banda utilizada.

\subsubsection{Integração GOES}

Os dados do GOES são fornecidos no Tempo Universal Coordenado (em inglês, Universal Coordinated Time -UTC) e, dada a extensão territorial de alguns biomas, como a Amazônia que engloba 3 fusos horários, há a necessidade de convertê-los para a hora local (HL).

Foi estimado a partir dos dados GOES de 1997 a 2015 em HL a densidade de FRP (FRP goes $(l o n+$ $x$, lat $+y$ ) para cada ponto da grade a partir de uma máscara de convolução $n x, y$ de $8 \mathrm{~km}$ x $8 \mathrm{~km}$, que resultou no agrupamento dos focos de queimadas e seus respectivos valores de FRP em uma grade para o passo de tempo de $15 \min \left(F R P_{\left.\text {grade }_{(l o n, l a t, t}\right)}\right)$, expresso na Equação 4.

$$
F R P_{\text {grade }_{(\text {lon }, \text { lat }, t)}}=\sum_{x=-\alpha}^{\alpha} \sum_{y=-\beta}^{\beta} n(x, y) F R P_{\text {goes }}(\text { lon }+x, \text { lat }+y, t)
$$

A grade resultante é definida para todos os pontos na qual a máscara de tamanho $\mathrm{M} \times \mathrm{N}$ sobrepõem completamente a imagem da área de estudo. Nesta etapa, os dados saturados do GOES corrigidos foram incluídos na análise.

Os arquivos resultantes da Equação 4 foram agrupados para cada dia da série temporal (1997-2015) para verificar a evolução temporal dos focos de queimadas durante 24 horas e o tipo de uso e cobertura da terra correspondente.

Com a finalidade de gerar histogramas com a frequência de observações do tempo de duração das queimadas, os histogramas da distribuição de frequências dos focos de queimadas ao longo do dia para a série temporal foram agrupados de acordo com o uso e cobertura da terra. 
Os histogramas com a frequencia de observações do tempo de duração das queimadas foram ajustados a partir de uma gaussiana utilizando a Equação 5 através de um programa em Interactive Data Language (IDL).

$$
\begin{array}{r}
f(x)=A_{0} e^{\frac{-Z^{2}}{2}} \\
z=\frac{X-A_{1}}{A_{2}}
\end{array}
$$

em que $A_{0}$ é a altura da Gaussiana, $A_{1}$ é o centro da Gaussiana, $A_{2}$ é o desvio padrão. Com a finalidade de comparar a distribuição da amostra observada com a distribuição de probabilidade esperada utilizou-se um teste Qui-Quadrado $\left(X^{2}\right)$ descrito como (Equação 7):

$$
x^{2}=\sum \frac{(O-E)^{2}}{E}
$$

onde $O$ é a frequência observada para cada classe e $E$ a frequência esperada para cada classe.

Se as frequências observadas diferirem significativamente das frequências esperadas, os valores obtidos no teste qui-quadrado serão grandes e, por conseguinte, o ajuste será ruim. Essa situação exige a rejeição da hipótese de que as frequências observadas são uma aproximação precisa da distribuição de frequência esperada (H0).

Se a H0 é aceita, gera um arquivo contendo o histograma ajustado para uma gaussiana com a frequência de observações do tempo de duração das queimadas e o uso do solo associado. A média de duração das queimadas é associado ao produto MCD12Q1 reclassificado para os anos de 2001-2013.

\subsubsection{Implementação no 3BEM_FRP.}

No 3BEM_FRP, a partir da assimilação da FRP estima-se a FRE e, por conseguinte, a emissão associada. A FRP estimada pelos diferentes sensores $\xi$ (lon, lat) em um determinado intervalo de tempo $(t)$ é agrupada em uma grade $\left(F R P_{\text {grid }}\right)$, resultante da convolução de uma máscara $\eta(\gamma, k)$, de tamanho $\mathrm{M} \mathrm{x} \mathrm{N}$ (colunas $\mathrm{x}$ linhas) sobre os valores de FRP (Equação 8).

$$
F R P_{\operatorname{grid}_{(l o n, l a t, t)}}=\sum_{\gamma=-a}^{\alpha} \sum_{\kappa=-b}^{\beta} \eta(\gamma, k) \xi(\text { lon }+\gamma, \text { lat }+\kappa, t)
$$

Deste modo, através da estimativa da FRP e seus respectivos horários de ocorrência para cada ponto de grade, a FRE é estimada pela seguinte fórmula (Equação 9):

$$
F R E_{\text {grid }_{(\text {lon }, \text { lat }, t)}}=\frac{1}{2} \sum_{i=1}^{n}\left(F R P_{n}+F R P_{n+1}\right)\left(T_{n+1}-T_{n}\right)
$$

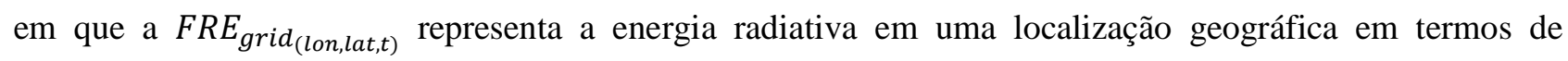
longitude e latitude de um ponto centrado em uma grade regular, $T$ o intervalo entre as observações e $n$ representa a enésima observação. Nesta etapa, se o número de observações de pixels de calor for igual a 1, o tempo total de queima corresponderá à média do ciclo diurno das queimadas associadas ao produto MCD12Q1 reclassificado para os anos de 2001-2013. Ressalta-se que para os anos posteriores à 2013, utiliza-se o mapa deste ano, uma vez que a análise do ciclo diurno das queimadas foi elaborada para a coleção 5 do produto, que contempla apenas de 2001 a 2013.

Durante a integração dos valores de FRP, se o intervalo de tempo entre as observações for maior que 4 horas, considera a existência de uma nova queima, e uma nova integração dos dados é iniciada. O modelo 3BEM_FRP considera que a queimada inicia uma hora e meia antes do primeiro foco e finaliza uma hora e meia após o último foco, acrescentando esse tempo a integração da FRE. 
A massa de gás emitido é expressada pela Equação 10, em que FEER corresponde ao produto Fire Energetics and Emissions Research version 1.0 (FEER.v1) em (kg/MJ) (ICHOKU; ELLISON, 2014) e EF aos fatores de emissão de Andreae e Merlet (2001) e YOKELSON et al. (2013) por espécie.

$$
M^{[\epsilon]}=F R E_{\text {grid }_{(l o n, l a t, t)}} \times F E E R \times \frac{E F^{[\epsilon]}}{E F^{[T P M]}}
$$

O banco de dados de queimadas utilizado pelo 3BEM_FRP é uma combinação do algoritmo WFABBA/GOES e dos produtos MOD14 e MYD14 do sensor MODIS. Deste modo, os valores anuais de emissões recorrentes da queima de biomassa obtidos através da inclusão dos valores do ciclo diurno das queimadas no 3BEM_FRP (C3BEM_FRP) foram analisados espacialmente entre 2003 e 2020.

\section{Resultados}

\subsection{Estimativa do ciclo diurno das queimadas}

O tempo médio de duração das queimadas para o ano de 2003 é apresentado na Figura 2. Para o período de análise, de 2001 a 2013, cerca de $61 \%$ das queimadas na América do Sul duram em média entre 6 e 7 horas, enquanto que $17 \%$ duram em média entre 7 e 8 horas. Enquanto que aproximadamente 12\% duram menos que 5 horas e $9 \%$ duram mais que 9 horas.

No bioma Floresta Tropical e Subtropical (Figura 1), localizado na região da Amazônia, a classe Floresta Ombrófila Densa que ocupa cerca de $80 \%$ do bioma tem queimadas que duram em média 6 horas, enquanto que a mesma classe localizada no bioma Savanas queima cerca de 30 minutos a mais. Neste bioma, as classes Gramíneas, Predominância Agrícola, Mosaico de áreas agrícolas/ Vegetação duram em média 6,75, 6,73 e 6,07 horas, respectivamente.

No bioma Savanas as classes predominantes, Savana Lenhosa e Savana queimam em média 6,29, e 6,31, horas. A classe Gramíneas, localizada principalmente no Rio Grande do Sul e Uruguai, queima em média 7,13 horas, cerca de 22 min a mais que a mesma classe situada na região da Amazônia. Ao passo que o tempo médio de duração das classes Predominância agrícola e Mosaico de Áreas Agrícolas e Vegetação no bioma Savanas se assemelham aos encontrados no bioma Floresta Tropical e Subtropical, queimando em média 6,83 e 6,88 , respectivamente.

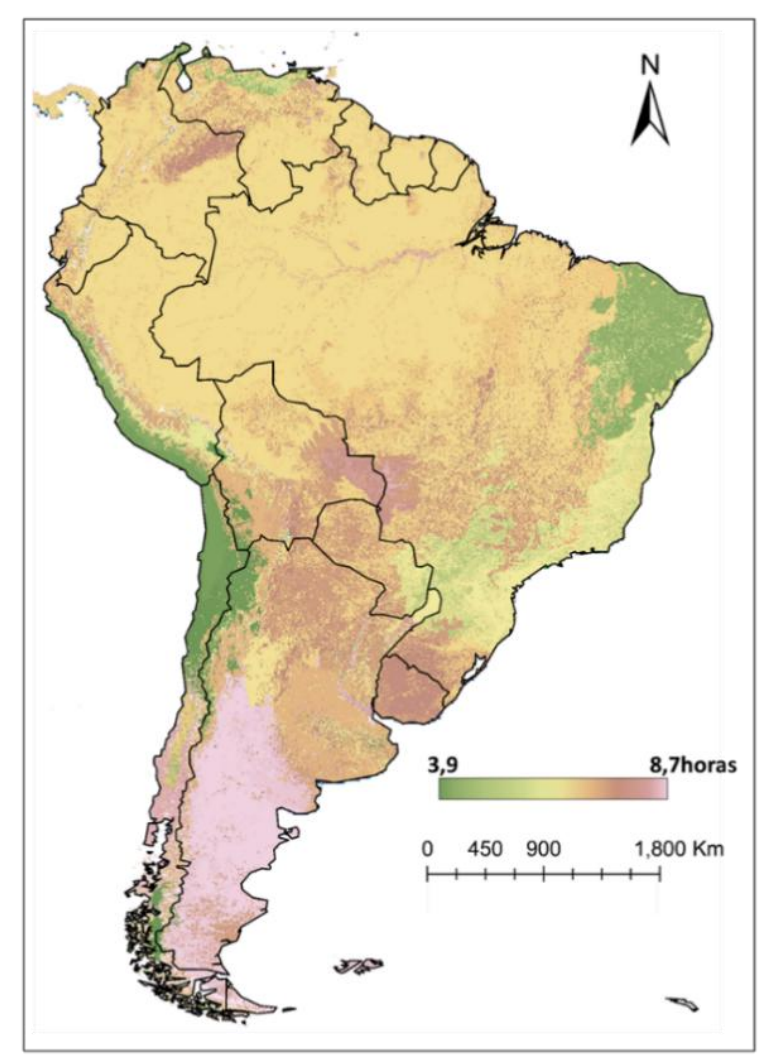

Figura 2: Distribuição da duração média das queimadas para a América do Sul para 2003. 
Na Mata Atlântica, a classe Floresta Ombrófila Densa queima em média 5,96 aproximando dos valores encontrados para a mesma classe no bioma Floresta Tropical e Subtropical. A classe Savana, encontrada principalmente nas áreas limítrofe com o bioma Floresta Tropical e Subtropical úmida, queimam em média 5,89 horas. Nesse bioma, as classes Predominância agrícola e Mosaico de Áreas Agrícolas e Vegetação queimam em média 6,35, 5,30 e 5,62, nesta ordem.

O bioma Caatinga é majoritariamente coberto pela classe Savanas, que queimam em média 4,70. Dentre os biomas brasileiros, as classes Gramíneas, Mosaico de Áreas Agrícolas e Vegetação e Predominância agrícola na Caatinga possuem o menor tempo médio de duração, com queimadas que duram em média 4,63, 4,16 e 4,76 horas, respectivamente.

O tempo médio de duração das queimadas no bioma Gramíneas Temperadas, Savanas e Vegetação Arbustiva, localizada ao sul da Argentina, são os maiores da América do Sul. As classes predominantes, Vegetação Arbustiva Fechada e Vegetação Arbustiva Aberta queimam em média 8,37 e 8,53 horas, respectivamente. Neste bioma, as Gramíneas queimam em média 6,94, enquanto que nas áreas de Predominância agrícola há a ocorrência de queimadas que duram em média 6,64 horas.

A classe Floresta Estacional Decidual está situada na Bolívia no bioma Savanas Estépica, em uma região adjacente ao Pantanal, com queimadas que duram em média 7,30 h. Nesse bioma, a classe Savana Lenhosa queima em média 6,95 horas.

\subsection{Análise da distribuição espacial de PM2.5 para a América do Sul estimados pelo C3BEM_FRP}

As Figuras 3, Figura 4 e Figura 5 exibem a distribuição espacial das emissões de Material Particulado (PM2.5) em teragramas para a América do Sul de 2003 a 2020 com a inclusão do ciclo diurno nas estimativas. Em geral, para toda a série histórica, o arco do desmatamento, localizado na região limítrofe entre o Cerrado e a Amazônia, apresentou os maiores registros de emissões de PM2.5. De acordo com CHEN (2013), dados obtidos por satélites mostram que o número de queimadas na Amazônia está positivamente relacionado com as taxas de desmatamento.

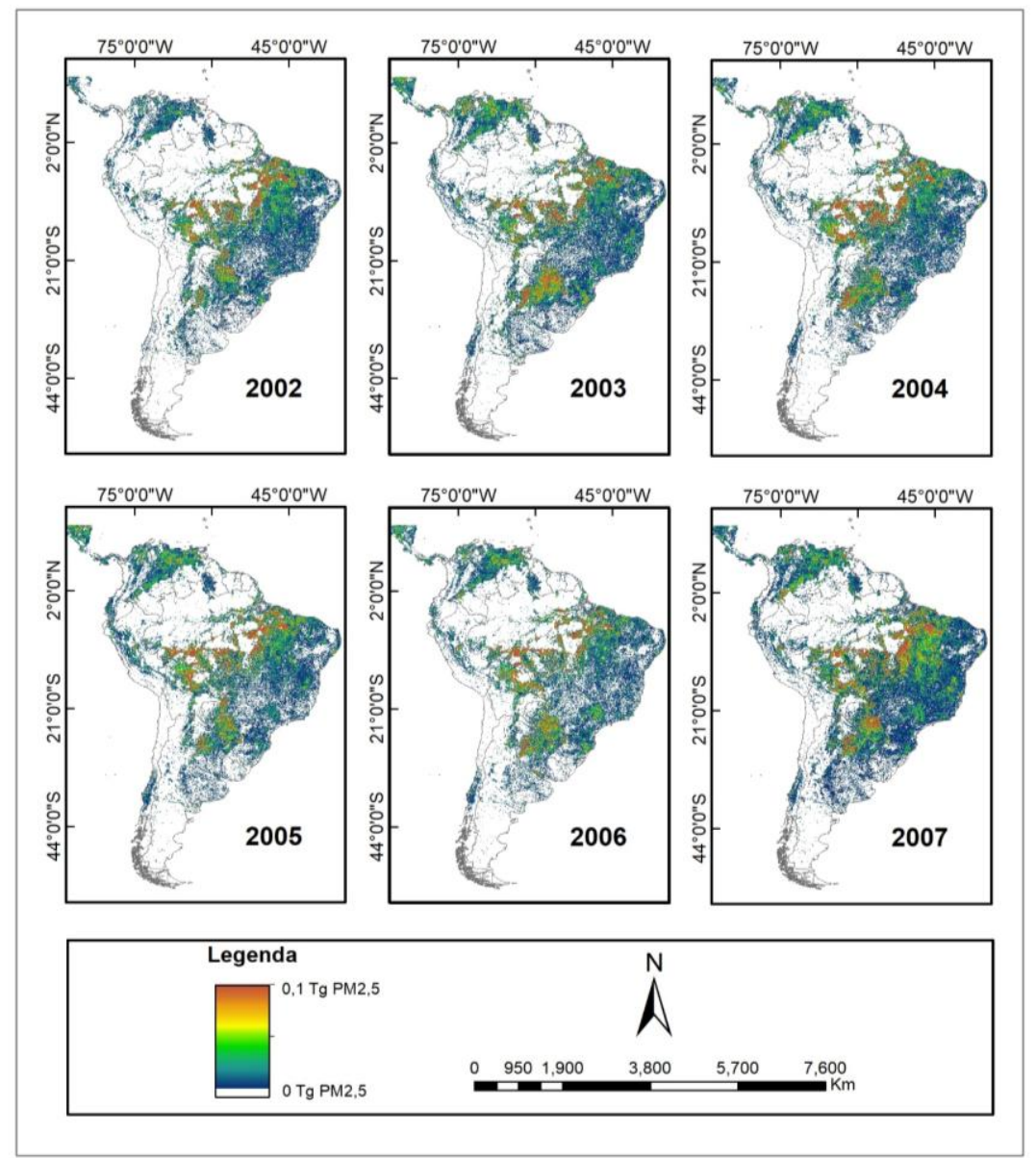

Figura 3: Distribuição espacial das estimativas emissões de PM2.5 para a América do Sul de 2002 a 2007. 


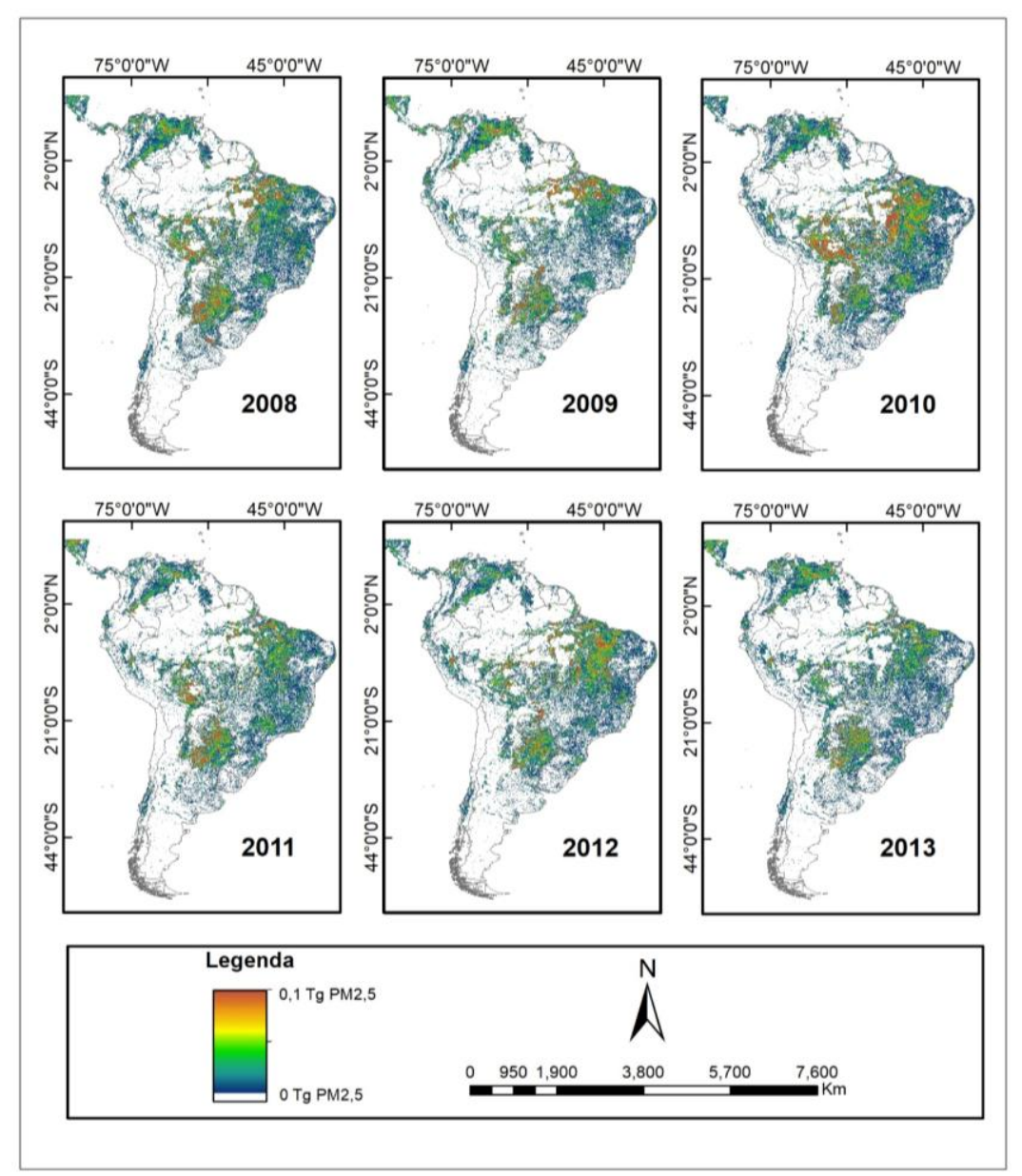

Figura 4: Distribuição espacial das estimativas emissões de PM2.5 para a América do Sul de 2008 a 2013.

Na região central da Amazônia não há ocorrência de emissões por ser uma região pouco habitada, onde dificilmente há a ignição natural de uma queimada, uma vez que altos indices de umidade e as copas densas tormam a floresta resistente a propagação do fogo. No entanto, a exploração florestal impulsionada pelas atividades humanas pode deixar a floresta mais inflamável, surgindo um regime do fogo resultante tanto das mudanças climáticas como do manejo da terra (COCHRANE; BARBER, 2009).

Dentre os países da América do Sul, o Brasil é o pais que mais emite PM2.5 oriundos da queima de biomassa, correspondendo a aproximadamente 58\% das emissões totais (2003-2020) para o continente. Para todos os anos em análise, as emissões de PM2.5 no território brasileiro superaram o somatório das emissões dos demais países.

Os estados brasileiros que mais emitem PM2.5 são o Pará, Mato Grosso, Maranhão, Rondônia, Tocantins e Amazonas, respectivamente (Figura 6). Durante os anos em análise, o Pará emitiu em média 1,2 Tg PM2.5 ano-1 com emissões associadas principalmente a porção norte do estado. As emissões deste estado ao longo da série temporal superam as emissões de países como a Argentina, Bolívia e Paraguai, que emitiram em média 0,9 Tg PM2.5 ano-1, 0,6 Tg PM2.5 ano $^{-1}$ e 0,5 Tg PM2.5 ano ${ }^{-1}$, nesta ordem. O segundo estado com os maiores registros de PM2.5 foi o Mato Grosso, cujas emissões corresponderam em média a 0,9 Tg PM2.5 $a^{-1}$. De acordo com o PRODES, Pará e Mato Grosso são os estados com as maiores taxas de desmatamento e, segundo Reddington (2015), as emissões de PM no Brasil possuem uma correlação positiva com as taxas anuais de desmatamento mapeadas pelo PRODES $(r=0,68$ a 0,97, $\mathrm{P}<0,05)$. 


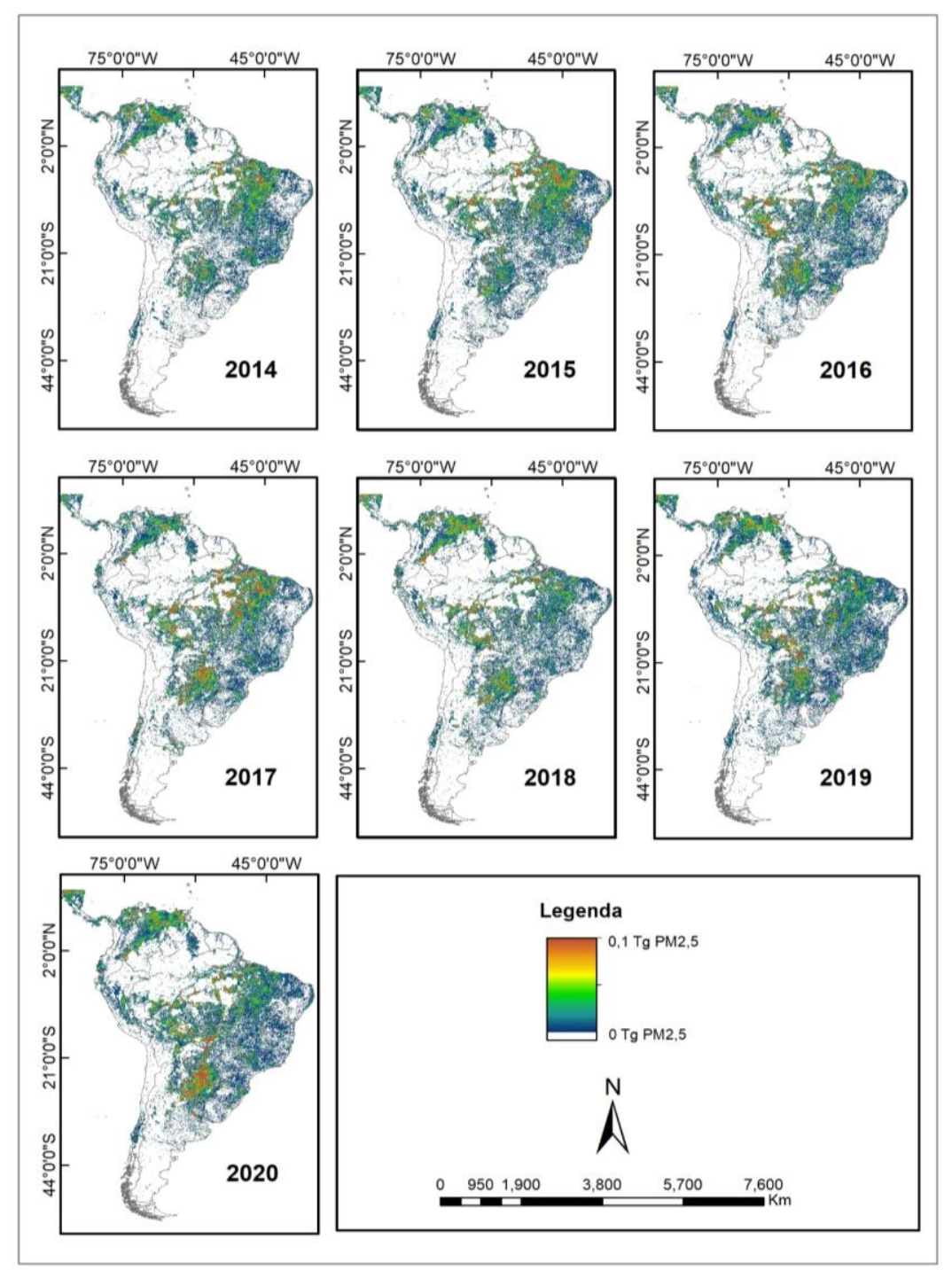

Figura 5: Distribuição espacial das estimativas emissões de PM2.5 para a América do Sul de 2014 a 2020.

Os estados com os maiores registros de emissão de PM2.5 na região sudeste foram Minas Gerais e São Paulo. Minas Gerais emitiu em média 0,2 Tg PM2.5 ano ${ }^{-1}$, com maior concentração de emissões associadas as regiões do Vale do Mucuri e Jequitinhonha. Nesta região há uma expansão da atividade silvicultural uma vez que o carvão vegetal é principal fonte de energia para produzir o aço no Brasil (LEITE; ALMEIDA; SILVA, 2012). Enquanto as emissões em São Paulo corresponderam em média a $0,08 \mathrm{Tg}$ PM2.5 ano ${ }^{-1}$. São Paulo é o maior produtor de cana-de-açúcar do Brasil, e embora seja comum durante a colheita queimar a palhada para facilitar a colheita, esta prática está diminuindo nos últimos anos com novas legislações vigentes (MATAVELI, 2014).

Piauí e Bahia foram os estados do Nordeste que apresentaram maiores taxas de emissões de PM2.5, ambos contabilizaram em média $0,2 \mathrm{Tg}$ PM2.5 ano $^{-1}$. A distribuição espacial das emissões nestes estados está relacionada a porção central do Piauí e na região do MATOPIBA. Nesta região, políticas públicas voltadas para a modernização agrícola aliadas a necessidade de expansão da fronteira agrícola fazem com que haja uma maior conversão da sua cobertura vegetal para estes fins. Deste modo, o fogo é utilizado para remover a vegetação natural e para o manejo das culturas, seja para queimar resíduos ou estimular a rebrota para a forragem (PIVELLO, 2011).

Embora o Brasil seja o país que mais contribui para o total de emissões para a América do Sul, a distribuição espacial das emissões indica uma concentração de PM2.5 principalmente no sul da Bolívia, norte da Argentina e oeste do Paraguai. A vegetação predominante nesta região é a floresta estacional decidual na região do Chaco, que queima em média 6 horas. Esta região é quente e seca e assim como o Cerrado é propensa a queimadas tanto de origem natural como antrópica. Sistemas agrícolas modernos avançaram sobre esta região e constituem uma ameaça aos ecossistemas naturais do Chaco. 


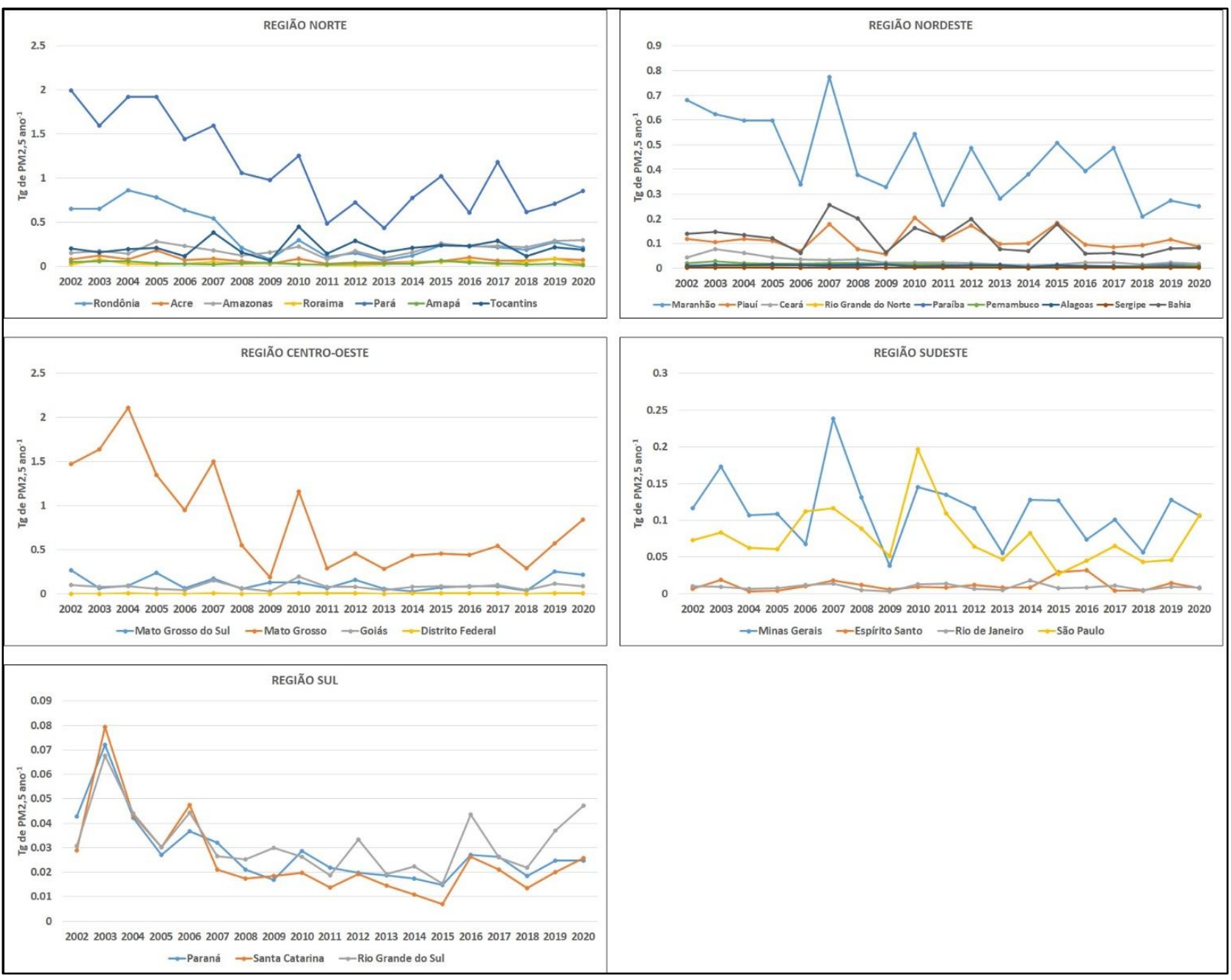

Figura 6: Emissão de PM2.5 por estado Brasileiro de 2003 a 2020.

\section{Conclusão}

Neste trabalho, foi desenvolvido uma metodologia acoplada ao modelo 3BEM_FRP que utiliza informações do tempo de duração das queimadas para diferentes tipos de uso e cobertura da terra para biomas da América do Sul com a finalidade de fornecer informações para a estimativa da FRE quando houver ausência de dados de FRP. Para a América do Sul, o MODIS pode ter até 4 aquisições diárias, o que é insuficiente para caracterizar o tempo de duração de uma queimada e, por conseguinte, subestimar as estimativas de FRE. Além disto, a cobertura de nuvens frequente na Amazônia dificulta a aquisição de dados fazendo com que a inclusão do ciclo colabore para melhorar as estimativas nesta região.

As informações do tempo médio de duração das queimadas para cada classe de uso de cobertura da terra do IGBP foram inseridas nos mapas da coleção 5 do produto MCD12Q1 de 2001 a 2013. O mapa do tempo médio de duração das queimadas 2013 serve como referência para gerar inventários após esse ano, o que é uma limitação do método e pode constituir uma fonte de incerteza. Sugere-se a atualização destes mapas para versão 6 do produto MCD12Q1 que contempla a série histórica de 2001 a 2019.

O modelo C3BEM_FRP considera que a queimada inicia uma hora e meia antes do primeiro foco e finaliza uma hora e meia após o último foco. Para estudos futuros sugere-se uma análise sobre o impacto desta inferência nas estimativas das emissões. Também sugere-se a realização de uma análise do impacto da resolução da grade na integração da FRP e consequentemente nas estimativas das emissões. Além disto, espera-se utilizar o C3BEM_FRP ciclo para assimilar as emissões de gases traços e aerossóis no modelo CCATT-BRAMS.

Por fim, pode-se concluir que o modelo C3BEM_FRP apresentou resultados consistentes e sua maior vantagem é viabilizar as estimativas de emissão quando há falta de informações do ciclo diurno da FRP. Além disso, o modelo também pode ser adaptado e inventários de emissões podem ser gerados por outros satélites como, por exemplo, o VIIRS e METEOSAT. 


\section{Referências}

BOWMAN, D. M. J. S. et al. Fire in the Earth System. Science, v. 481, n. 2009, p. 481-485, 2010.

CARMENTA, R. et al. Does the establishment of sustainable use reserves affect fire management in the humid tropics. PLoS ONE, v. 11, n. 2, p. 1-19, 2016.

CHEN, Y. et al. Long-term trends and interannual variability of forest, savanna and agricultural fires in South America. Carbon Management, v. 4, n. 6, p. 617-638, 2013.

COCHRAne, M. A. Tropical Fire Ecology: Climate Change, Land Use and Ecosystem Dynamics. Chichester, UK: Praxis Publishing, 2009.

COCHRANE, M. A.; BARBER, C. P. Climate change, human land use and future fires in the Amazon. Global Change Biology, v. 15, n. 3, p. 601-612, 2009.

FANIN, T.; WERF, G. R. VAN DER. Relationships between burned area, forest cover loss, and land cover change in the Brazilian Amazon based on satellite data. Biogeosciences, v. 12, n. 20, p. 6033-6043, 2015.

FREITAS, S. R. et al. Emissões de queimadas em ecossistemas da América do Sul. Estudos Avançados, v. 19, n. 53, p. 167-185, 2005.

FRIEDL, M. A. et al. Global land cover mapping from MODIS: algorithms and early results. Remote Sensing Of Environment, v. 83, p. 287-302, 2002.

GIGLIO, L. et al. The Collection 6 MODIS burned area mapping algorithm and product. Remote Sensing of Environment, v. 217, n. August, p. 72-85, 2018.

GILMAN, J. B. et al. Biomass burning emissions and potential air quality impacts of volatile organic compounds and other trace gases from fuels common in the US. Atmospheric Chemistry and Physics, v. 15, n. 24, p. 13915-13938, 2015.

HEILMAN, W. E. et al. Wildland fire emissions, carbon, and climate: Plume rise, atmospheric transport, and chemistry processes. Forest Ecology and Management, v. 317, p. 70-79, 2014.

ICHOKU, C. et al. Fire and Smoke Remote Sensing and Modeling Uncertainties: Case Studies in Northern Sub-Saharan Africa. Natural Hazard Uncertainty Assessment: Modeling and Decision Support, p. 215230, 2016.

ICHOKU, C.; ELLISON, L. Global top-down smoke-aerosol emissions estimation using satellite fire radiative power measurements. Atmospheric Chemistry and Physics, v. 14, n. 13, p. 6643-6667, 2014.

ICHOKU, C.; KAHN, R.; CHIN, M. Satellite contributions to the quantitative characterization of biomass burning for climate modeling. Atmospheric Research, v. 111, p. 1-28, 2012.

KAISER, J. W. et al. Biomass burning emissions estimated with a global fire assimilation system based on observed fire radiative power. Biogeosciences, v. 9, n. 1, p. 527-554, 2012.

KOLTUNOV, A.; USTIN, S. L.; PRINS, E. M. On timeliness and accuracy of wildfire detection by the GOES WF-ABBA algorithm over California during the 2006 fire season. Remote Sensing of Environment, v. 127, p. 194-209, 2012.

LEITE, M. E.; ALMEIDA, J. W. L.; SILVA, R. F. Análise espaço-temporal do eucalipto no Norte de Minas Gerais nos anos de 1986, 1996 e 2010. GeoTextos, v. 8, n. 2, p. 59-74, 2012.

LONGO, K. M. et al. The coupled aerosol and tracer transport model to the brazilian developments on the regional atmospheric modeling system (catt-brams)-part 2: Model sensitivity to the biomass burning inventories. Atmospheric Chemistry and Physics, v. 10, n. 13, p. 5785-5795, 2010.

LOVELAND, T. R.; BELWARD, A. S. The igbp-dis global 1km land cover data set, discover: First results. 
International Journal of Remote Sensing, v. 18, n. 15, p. 3289-3295, 1997.

MATAVELI, G. A. V. Análise da influência do ângulo de visada e do background na estimativa da potência radiativa do fogo. [s.l.] Instituto Nacional de Pesquisas Espaciais, 2014.

OLSON, D. M. et al. Terrestrial Ecoregions of the World: A New Map of Life on Earth. BioScience, v. 51, n. 11, p. 933, 2001.

PEREIRA, G. et al. Estimating trace gas and aerosol emissions over South America: Relationship between fire radiative energy released and aerosol optical depth observations. Atmospheric Environment, v. 43, n. 40, p. 6388-6397, 2009.

PEREIRA, G. et al. Estimativa e assimilação das emissões de gases traços e aerossóis de queimadas em modelos de química atmosférica. [s.l.] Instituto Nacional de Pesquisas Espaciais, 2013.

PEREIRA, G. et al. Assessment of fire emission inventories during the South American Biomass Burning Analysis (SAMBBA) experiment. Atmospheric Chemistry and Physics, v. 16, n. 11, p. 6961-6975, 2016.

PIVELLO, V. R. The use of fire in the cerrado and Amazonian rainforests of Brazil: Past and present. Fire Ecology, v. 7, n. 1, p. 24-39, 2011.

PROSPERI, P. et al. New estimates of greenhouse gas emissions from biomass burning and peat fires using MODIS Collection 6 burned areas. Climatic Change, v. 161, n. 3, p. 415-432, 2020.

QUÉRÉ, C. LE et al. Global Carbon Budget 2015. Earth System Science Data, v. 7, n. 2, p. 349-396, 2015.

REBOITA, M. S. et al. Regimes de precipitação na América do Sul: uma revisão bibliográfica. Revista Brasileira de Meteorologia, v. 25, n. 2, p. 185-204, 2010.

REDDINGTON, C. L. et al. Air quality and human health improvements from reductions in deforestationrelated fire in Brazil. Nature Geoscience, v. 8, n. 10, p. 768-771, 2015.

REID, J. S. et al. Global monitoring and forecasting of biomass-burning smoke: Description of and lessons from the fire Locating and Modeling of Burning Emissions (FLAMBE) program. IEEE Journal of Selected Topics in Applied Earth Observations and Remote Sensing, v. 2, n. 3, p. 144-162, 2009.

SOMMERS, W. T.; LOEHMAN, R. A.; HARDY, C. C. Wildland fire emissions, carbon, and climate: Science overview and knowledge needs. Forest Ecology and Management, v. 317, p. 1-8, 2014.

WERF, G. R. VAN DER et al. Global fire emissions estimates during 1997-2015. Earth System Science Data Discussions, n. January, p. 1-43, 2017.

WIEDINMYER, C. et al. The Fire INventory from NCAR (FINN) - a high resolution global model to estimate the emissions from open burning. Geoscientific Model Development Discussions, v. 3, n. 4, p. 2439-2476, 2010.

WOOSTER, M. J. et al. Retrieval of biomass combustion rates and totals from fire radiative power observations: FRP derivation and calibration relationships between biomass consumption and fire radiative energy release. Journal of Geophysical Research Atmospheres, v. 110, n. 24, p. 1-24, 2005.

WOOSTER, M. J.; ZHUKOV, B.; OERTEL, D. Fire radiative energy for quantitative study of biomass burning: Derivation from the BIRD experimental satellite and comparison to MODIS fire products. Remote Sensing of Environment, v. 86, n. 1, p. 83-107, 2003.

XU, W. et al. New GOES imager algorithms for cloud and active fire detection and fire radiative power assessment across North, South and Central America. Remote Sensing of Environment, v. 114, n. 9, p. 1876-1895, 2010.

YOKELSON, R. J. et al. Coupling field and laboratory measurements to estimate the emission factors of 
identified and unidentified trace gases for prescribed fires. Atmospheric Chemistry and Physics, v. 13, n. 1, p. 89-116, 2013.

(i) (2) (2)

Este artigo é distribuído nos termos e condições do Creative Commons Attributions/Atribuição-

BY NãoComercial-CompartilhaIgual (CC BY-NC-SA). 